\title{
AN IMPROVED AUTONOMOUS EXPLORATION FRAMEWORK FOR INDOOR MOBILE ROBOTICS USING REDUCED APPROXIMATED GENERALIZED VORONOI GRAPHS
}

\author{
Xinkai Zuo ${ }^{1}$, Fan Yang ${ }^{1}$, Yifan Liang ${ }^{1}$, Zhou Gang ${ }^{1}$, Fei Su ${ }^{1}$, Haihong Zhu ${ }^{1}$, Lin Li ${ }^{1,2}$ * \\ ${ }^{1}$ School of Resource and Environmental Sciences, Wuhan University, 129 Luoyu Road, Wuhan 430079, China; \{zuoxinkai2012, \\ yhlx125, lyf0312, 2014301130059, sftx016, hhzhu, lilin\}@whu.edu.cn; \\ ${ }^{2}$ Collaborative Innovation Centre of Geospatial Technology, Wuhan University, 129 Luoyu Road, Wuhan 430079, China
}

Comission I, ICWG I/IV

KEY WORDS: Autonomous Robotic Exploration, Weak Edges, Reduced Approximated GVG

\begin{abstract}
:
In the field of autonomous navigation for robotics, one of the most challenging issues is to locate the Next-Best-View and to guide robotics through a previously unknown environment. Existing methods based on generalized Voronoi graphs (GVGs) have presented feasible solutions but require excessive computation to construct GVGs from metric maps, and the GVGs are usually redundant. This paper proposes a reduced approximated GVG (RAGVG), which provides a topological representation of the explored space with a smaller graph. To be specific, a fast and practical algorithm for constructing RAGVGs from metric maps is presented, and a RAGVGbased autonomous robotic exploration framework is designed and implemented. The proposed method for constructing RAGVGs is validated with two known common maps, while the RAGVG-based autonomous exploration framework is tested on two simulation and one real-world museum. The experimental results show that the proposed algorithm is efficient in constructing RAGVGs, and indicate that the mobile robot controlled by the RAGVG-based autonomous exploration framework, compared with famous frontiersbased method, reduced the total time by approximately $20 \%$ for the given tasks.
\end{abstract}

\section{INTRODUCTION}

One of the most challenging issues in mobile robotics is the ability to autonomously explore previously unknown spaces (Burgard, Moors, Stachniss, et al, 2005). With the help of an exploration strategy, mobile robots can autonomously decide where to go and build an environment map of a previously unknown space to accomplish a given task, such as map building (Stachniss, Burgard, 2003), search and rescue (Calisi, Farinelli, Iocchi et al, 2005), and 3D model building (Low, Lastra, 2006; Quintana, Prieto, Adán et al, 2016). Hence, a good exploration strategy will enable a mobile robot to cover a space completely in an acceptable amount of time (Grisetti, Stachniss, Burgard, 2007).

According to existing research, many clever methods have been proposed and successfully implemented, and the most common solutions are based on Next-Best-View (NBV) (Kulich, Kubalík, Přeučil, 2019; González-Banos, Latombe, 2002). The well-known frontier-based methods (Yamauchi, 1997; Visser, Van Ittersum, Jaime et al, 2007) are typical NBV-based solutions. Nevertheless, due to the complexity of metric maps, frontierbased methods suffer from low efficiency in evaluating candidate points (CPs) (Tsardoulias, Iliakopoulou, Kargakos et al, 2017) and planning global paths (Thrun, 1998). To avoid the complexity of metric maps, topological maps are employed to model the environment. The generalized Voronoi graph (GVG) is a kind of topological map, and it performs well as a basis for sensor-based path planning in an unknown static environment (Choset, 1995). However, it remains difficult because the existing algorithms for constructing GVGs are usually complex and unstable (Nagatani, Choset, 1999; Choset, 2000).

In this paper, we propose a straightforward and stable method for constructing a reduced approximated GVG (RAGVG) from an occupancy grid map (OGM) to represent the indoor environments and design an autonomous robotic exploration system using RAGVGs to accelerate the decision-making process.

The remaining content of this paper is structured as follows. Section 2 reviews previous research and existing methods regarding autonomous robotics exploration and GVGs. Section 3 provides a detailed explanation of RAGVGs and the proposed algorithms for constructing RAGVGs. Section 4 introduces the workflow and the other necessary modules in the proposed RAGVG-based framework. Section 5 presents the experimental design and discusses the results of the simulations and the realworld experiment. In Section 6, we draw some conclusions about our research and discuss the prospects of autonomous robotic exploration.

\section{RELATED WORKS}

The mainstream approach regards exploration as an incremental NBV process. We focus on recent approaches based on frontiers and GVGs, respectively, since these have been the most successful and are most closely related to the proposed method. In addition, the common algorithms for constructing GVGs are also discussed.

\subsection{Frontier-based Exploration}

The frontier-based methods involve extracting frontiers between free space and unexplored space, and the NBV is selected from the CPs that are located on the centroids of the frontiers (Yamauchi, 1997). The main challenge of the frontierbased method is how to select the NBV from the CPs. In the earliest versions (González-Banos, Latombe, 2002; Visser, Van

\footnotetext{
* Corresponding author
} 
Ittersum, Jaime et al, 2007), a global utility function that linearly combines the distance and the estimation of information gain was proposed. Authors (Stachniss, Grisetti, Burgard, 2005) presented a decision-theoretic framework that considers the uncertainty and expected information gain to evaluate the cost of executing an action. Then, improved methods were proposed in the next few years. Basilico and Amigoni (Basilico, Amigoni, 2011) extended the global utility function using a fuzzy measure approach that linearly combined several criteria. Carrillo and colleagues (Carrillo, Dames, Kumar et al, 2015; Carrillo, Dames, Kumar et al, 2018) designed a utility function based on Shannon and Rényi entropy theory according to the estimation of potential information gain.

The frontier-based methods enjoy the advantage of easy implementation but suffer from low efficiency in evaluating CPs and low extensibility to various applications, especially in a large-scale space (Thrun, 1998). First, frontier-based methods usually generate a great number of CPs from many frontiers. As a result, most CPs are too meaningless and redundant so that a substantial amount of time is wasted on evaluating them. Second, to provide an important feature for evaluating CPs, all the global paths from the current location of the robot to each $\mathrm{CP}$ must be found. The heuristic algorithms (such as $\mathrm{A}^{*}$ ) can be helpful to find a path, but they are too inefficient to satisfy real-time requirements and cannot guarantee success when there are many scattered pixels in the OGM (Tsardoulias, Iliakopoulou, Kargakos et al, 2016; Valero-Gomez, Gomez, Garrido et al, 2013).

\subsection{GVG-based Navigation and Exploration}

In recent years, topological graphs have been the focus of robotic navigation studies. In particular, a few studies focus on developing navigation algorithms and autonomous robotics exploration frameworks on the basis of generalized Voronoi diagrams (GVDs) and GVGs. Takahashi and Schilling (Takahashi, Schilling, 1989) presented a novel method for mobile robotics motion planning in a plane using GVD. ValeroGomez et al. (Valero-Gomez, Gomez, Garrido et al, 2013) provided a comprehensive view of the Fast Marching algorithm and presented an efficient method for planning safer mobile robot trajectories using GVD. Tsardoulias et al. (Tsardoulias, Iliakopoulou, Kargakos et al, 2016) demonstrated that the path planning algorithms based on the GVD are faster and have a higher success rate, and the resulting paths are guaranteed to be collision-free. Choset and his team (Choset, 1995; Choset, 2000), by extending the GVD from one dimension to multi-dimension, proposed the GVG and the hierarchical GVG (HGVG) and proved them sufficient for motion planning. Nagatani and Choset (Nagatani, Choset, 1999) proposed an algorithm to reduce the unnecessary edges and nodes of GVGs and presented an exploration method using a reduced GVGs (RGVGs). Tsardoulias et al. (Tsardoulias, Iliakopoulou, Kargakos et al, 2017) compared three methods of target selection for full exploration of an unknown space based on approximated GVGs (AGVGs), and the experiments showed that the AGVGs can perform well in autonomous robotic exploration.

The most crucial step for constructing a GVG from an OGM is extracting GVD by means of image thinning algorithms, e.g., the brushfire algorithm (Choset, Lynch, Hutchinson et al, 2005; Lau, Sprunk, Burgard, 2010), the Zhang-Suen algorithm (Zhang, Suen, 1984) and morphology-based algorithms (Saeed, Rybnik, Tabedzki, 2001; Saeed, Tabędzki, Rybnik et al, 2010).

The GVG-based autonomous exploration methods have the advantages of high efficiency in decision making and better extensibility due to the topological representation of the explored space. Nevertheless, the complex steps for constructing
GVG can be simplified and improved. The main problem of the GVG-based methods is that the most of existing algorithms for constructing GVGs from OGMs are usually complex and unstable (Tsardoulias, Serafi, Panourgia et al, 2014). Because the GVDs extracted by existing image thinning algorithms are often cracked, redundant and disordered, some post-processing steps have to be conducted to eliminate unnecessary nodes and edges (Nagatani, Choset, 1999), which potentially leads to information loss. In addition, the autonomous exploration system requires relatively efficient algorithms to construct GVGs to satisfy realtime decision making. Thus, a fast and stable method for extracting qualified GVDs from OGMs is needed.

\section{CONSTRUCTION OF REDUCED APPROXIMATED GVGS}

The RAGVGs introduced in this section are exhaustive, nonredundant and non-interrupted. Therefore, the improvements attained are that (I) the resulting RAGVGs can be directly regarded as the topological maps of the explored environment, without any outlier elimination or other post-processing steps, and that (II) the RAGVGs can cover almost every corner of the explored spaces with relatively small graphs.

As illustrated in Figure 1, the proposed three-stage method for constructing RAGVGs from OGMs can be described as: (I) a few image pre-processing steps for extracting a smooth free area map, (II) the proposed corner rounding method and image thinning algorithm for generating an RAGVD and (III) a flood-fill algorithm for constructing the topological graph.

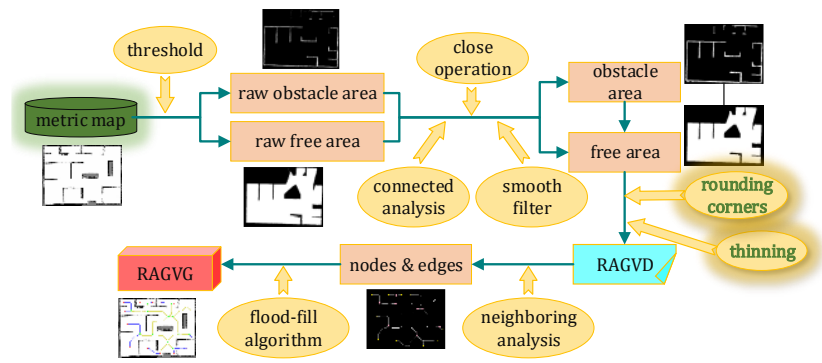

Figure 1. Workflow of constructing RAGVGs

\subsection{Pre-processing}

The OGM is a map of probabilities, so it cannot be directly used for indoor exploration and navigation. The free area map (FAM) represents the absolutely passable regions for mobile robots in the explored environment, e.g., at least $30 \mathrm{~cm}$ away from obstacles. An example of extracting the FAM from an OGM is shown in Figure 2. First, a grey value threshold $x \geq t_{1}$ is applied on the OGM; then, the small outliers are removed through a connected component analysis; finally, a buffer of the obstacle area, which is extracted by a grey value threshold $x \leq t_{2}$, is subtracted.
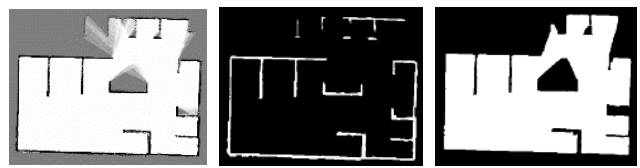

Figure 2. The OGM (left); The buffer of obstacle area (mid); (c) The free area map (right)

\subsection{Eliminating the Weak Edges}

3.2.1 Preliminary: Choset et al. (Choset, 1995) defined the GVD and the GVG by means of a distance function $d_{i}(x)$ and a 
distance gradient $\nabla d_{i}(x)$. Let two obstacles in the metric map be point sets $C_{i}$ and $C_{j}$, and the Two-Equidistant Face $\mathcal{F}_{i j}$ and the Two-Voronoi Set $\mathcal{F}^{2}$ can be termed as Eq.(1) and Eq.(2), respectively.

$$
\mathcal{F}_{i j}=\left\{\begin{array}{c}
x \in \mathbb{R}^{2} \mid d_{i}(x)=d_{j}(x), \nabla d_{i}(x) \neq \nabla d_{j}(x), \\
\forall k \neq i, j: d_{i}(x) \neq d_{k}(x)
\end{array}\right\},
$$

where $d_{i}(x)$ is the minimum distances among point $x$ and all points in obstacle set $C_{i}$, and gradient $\nabla d_{i}(x)$ is a unit vector in the direction from point $c_{0}$ to $x$, where $c_{0}$ is the nearest point to $x$ in $C_{i}$.

$$
\mathcal{F}^{2}=\bigcup_{i=1}^{n-1} \bigcup_{j=i+1}^{n} \mathcal{F}_{i j} .
$$

As is shown in Figure 3, $\mathcal{F}_{i j}$ is the set of points equidistant to $C_{i}$ and $C_{j}$, and $\mathcal{F}^{2}$ is the set of points equidistant to two or more obstacles. Hence, $\mathcal{F}^{2}$ can be regarded as the GVD of the 2D space that consists of obstacles set $\left\{C_{i}\right\}$.

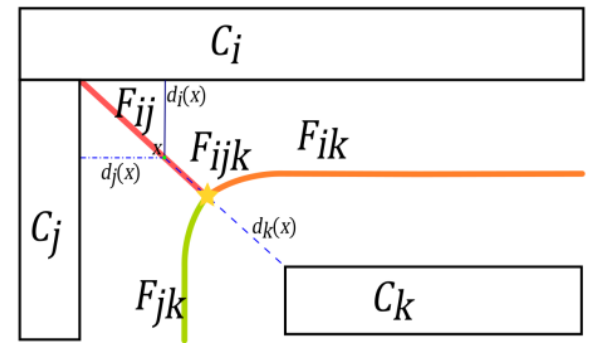

Figure 3. An example of a GVG.

The GVG is a topological graph constructed from the GVD. To define the GVG, the Three-Equidistant Face $\mathcal{F}_{i j k}$ and Three Voronoi Set $\mathcal{F}^{3}$ is defined as Eq.(3) and Eq.(4), respectively. In Figure $3, \mathcal{F}_{i j k}$ is the point equidistant to $C_{i}, C_{j}$ and $C_{k}$, which is also the joint of $\mathcal{F}_{i j}, \mathcal{F}_{j k}$ and $\mathcal{F}_{i k}$; then, $\mathcal{F}^{3}$ is the set of points equidistant to three or more obstacles. Hence, $\mathcal{F}^{3}$ can be regarded as the joints of the GVD.

$$
\begin{gathered}
\mathcal{F}_{i j k}=\mathcal{F}_{i j} \cap \mathcal{F}_{i k} \cap \mathcal{F}_{j k}=\mathcal{F}_{i j} \cap \mathcal{F}_{i k} . \\
\mathcal{F}^{3}=\bigcup_{i=1}^{n-2} \bigcup_{j=i+1}^{n-1} \bigcup_{k=j+1}^{n} \mathcal{F}_{i j k} . \\
\text { GVG }=\left\{\mathcal{F}^{2}, \mathcal{F}^{3}\right\} .
\end{gathered}
$$

With these definitions above, in a 2D space, the GVG can be defined as Eq.(5), where $\mathcal{F}^{2}$ denotes the set of Generalized Voronoi Edges and $\mathcal{F}^{3}$ represents the set of Generalized Voronoi Vertexes. Having edges and vertexes, the topological graph GVG can be constructed after computing its distance matrix.

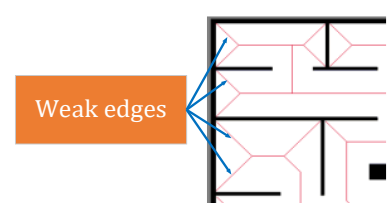

(a)

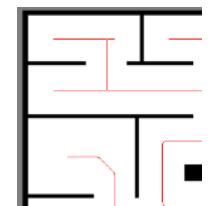

(b)
Figure 4. (a) A GVD; (b) A reduced approximated GVD.

However, the GVGs are usually redundant because weak edges exist at some concave walls (e.g., corners), as is depicted in Figure 4(a). These weak edges are unnecessary for robotic exploration and navigation, and they increase the size of the topological graph with information of low value. The RAGVG proposed in this paper is a topological graph constructed from a reduced approximation of GVD, as depicted in Figure 4(b). Without any weak edges, the RAGVGs consist of pivotal nodes and edges. Moreover, it is confirmed in our research that the RAGVGs can also preserve almost the same connectivity and coverage information as the original GVGs.

3.2.2 Corner Rounding Method: A few studies (Nagatani, Choset, 1999; Lau, Sprunk, Burgard, 2010; Tsardoulias, Serafi, Panourgia et al, 2014) noticed these redundant weak edges and designed some post-processing methods to eliminate weak edges from original GVGs. We investigate the reason why the weak edges occur, and propose a simple method for eliminating the weak edges.

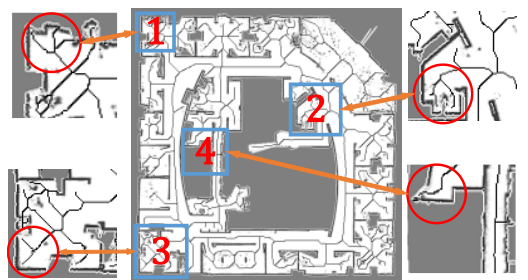

Figure 5. The reason for the occurrence of the weak edges, where "1" and " 2 " refer to rough wall surfaces, and " 3 " and " 4 " refer to concave wall corners.

We find that there are two conditions where exist weak edges, as shown in Figure 5. One is rough wall surface that leads to a number of local maximums of distance, and the other one is concave wall corner that leads to one local maximum of distance. In fact, two conditions can be summarized into one common reason, that is, concave areas where concave angles exist.

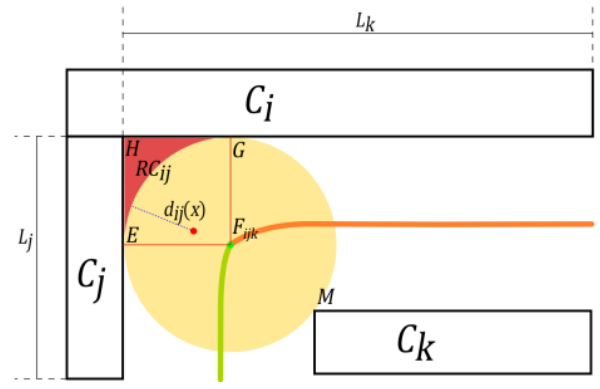

Figure 6 . Changing the mitre corner $\angle E H G$ to a rounded corner by adding $R C_{i j}$, which is shown by the red pixels.

To eliminate weak edges, we employ image processing operations to deal with these concave areas. For rough wall surfaces, it is quite easy to employ morphological operations to fill the holes and remove the bumps on the rough wall surfaces. For concave wall corners, the local maximum of distance can be eliminated by changing the mitre corner into rounded corner, and an example is shown in Figure 6.

The rounded corner is built by adding an obstacle $R C_{i j}=$ $\square E F_{i j k} G H-E F_{i j k} G$, where $E F_{i j k} G$ is a circular sector of a round face $R F$ termed as Eq.(6).

$$
R F=\left\{P \in \mathbb{R}^{2} \mid\left\|\mathcal{F}_{i j k}-P\right\| \leq d_{k}\left(\mathcal{F}_{i j k}\right)\right\} .
$$

It is easy to prove that no two-equidistant faces exist in $E F_{i j k} G$ because the minimum distance $d_{i j}(x)$ from any point $x \in$ $E F_{i j k} G$ to $R C_{i j}$ is unique. Hence, the weak edge $\mathcal{F}_{i j}$ is eliminated. Note that this elimination method can be applied to the concave wall corners with $\angle E H G<\pi$. 
However, it is difficult and time-consuming to locate point $\mathcal{F}_{i j k}$ and $H$ because of the complexity of the FAM. We thus have to look for another practical method to change mitre corners into rounded corners. We tested a number of smoothing filters and found that the combination of a median filter and an grey value threshold can approximately make all corner rounded. First, a median filter, whose size $s$ can be computed as Eq.(7), is applied on the whole FAM; then a threshold $t$, which is an empirical value, is used to retain the pixels $x \leq t$ to obtain a smoothed FAM. The local differences between a FAM and its smoothed version is shown in Figure 7.

$$
S=\left[\frac{3.0}{R}\right]+\left[\frac{3.0}{R}\right] \% 2
$$

where $R$, the resolution of the OGM, is defined as one pixel in the OGM represents an $R m \times R m$ region in the real-world, and note that $s$ must be odd so there exists an addition item $\left[\frac{3.0}{R}\right] \% 2$.

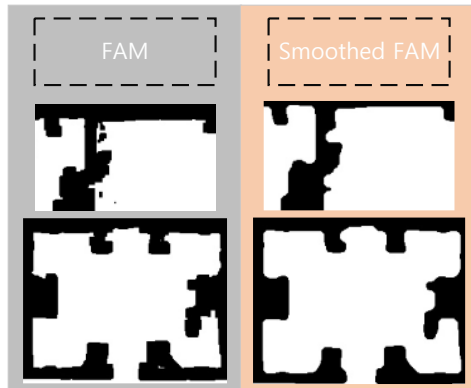

Figure 7. Parts of FAM (left) and smoothed FAM (right).

\subsection{Constructing RAGVGs}

KMM image thinning algorithm (Saeed, Rybnik, Tabedzki, 2001) can be applied to obtain an RAGVD from a SFAM without any rough wall surfaces or concave wall corners. The resulting RAGVDs are "approximated" because they does not strictly satisfy the rules about the distance transformation of GVD as mentioned above. Nevertheless, the RAGVDs are very close to GVDs, and this kind of approximation is provably sufficient for robotics navigation (Nagatani, Choset, 1999).

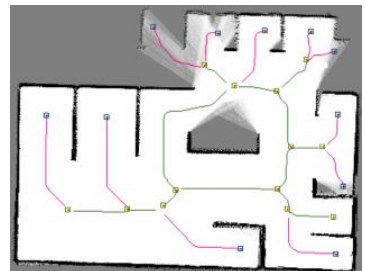

Figure 8. Four kinds of elements of an RAGVG.

To represent connectivity and accessibility, an RAGVD should be transformed into an RAGVG. An example of an RAGVG is shown in Figure 8. Four kinds of elements should be extracted: (1) a free endpoint should be transformed into an end, the degree of which is 1 ; (2) an intersection should be transformed into a joint, the degree of which is larger than 1; (3) an edge connecting an end with a related joint should be transformed into a branch and (4) an edge connecting two joints should be transformed into a link.

The approach of extracting four elements from the RAGVD is modified from the flood-fill algorithm, which is easy to determine and implement. Each end is bound to connect with one specific joint through a specific branch, and all links can roughly represent the primary structure, while all branches play a relatively unimportant role. Hence, it is reasonable to divide the RAGVG into surrounding network and main network. The set of all branches and their related ends and joints is regarded as the surrounding network. The sub-graph consisting of all links and their related joints is regarded as the main network. In addition, the distance matrices $D_{S}$ and $D_{M}$ according to the lengths of the branches and links are constructed as Eq.(8) and Eq.(9).

$$
\begin{gathered}
D_{S}^{i, j}=\left\{\begin{array}{c}
\text { lengt } h_{i, j}, \text { if } i \text {-th end is connected with } j \text {-th joint } \\
-1, \text { else }
\end{array},(8)\right. \\
D_{M}^{i, j}=D_{M}^{j, i}=\left\{\begin{array}{c}
\text { lengt } h_{i, j}, \text { if } i \text {-th joint connects with } j \text {-th joint } \\
-1, \text { else }
\end{array}\right.
\end{gathered}
$$

where length $h_{i, j}$ is a measure of how long the corresponding edge is, e.g., the number of pixel points.

\section{FULL-COVERAGE EXPLORATION USING RAGVGS}

The RAGVG-based autonomous exploration framework presented in this section transforms the problem representation from a Euclidean metric map into a topological graph space $\mathcal{G}=$ $\{E, V\}$. This framework is expected to make improvements that (III) CPs generated from an RAGVG are distinctly small in size, which leads to much less time consumption for decision making. In addition, (IV) graph-based path planning is extremely fast, and the global paths generated from RAGVGs are collision-free. Note that our research is for single robot exploration; however, the proposed method can be expanded to multi-robot collaboration conditions.

The whole workflow of the proposed RAGVG-based autonomous exploration framework is presented as Figure 9, and the steps are presented as follows:

Step (1): While the robot is moving, an OGM of the explored area is being updated in real time;

Step (2) An RAGVG is constructed for the current OGM;

Step (3) CPs are extracted from the nodes of the RAGVG;

Step (4) The CPs are evaluated by applying a Multi-Criteria Decision Making (MCDM) approach on some features to select the NBV;

Step (5) The robot is navigated to the NBV and the exploration strategy restarts from step (1) until there is no valid CP left after the filtering in step (3).

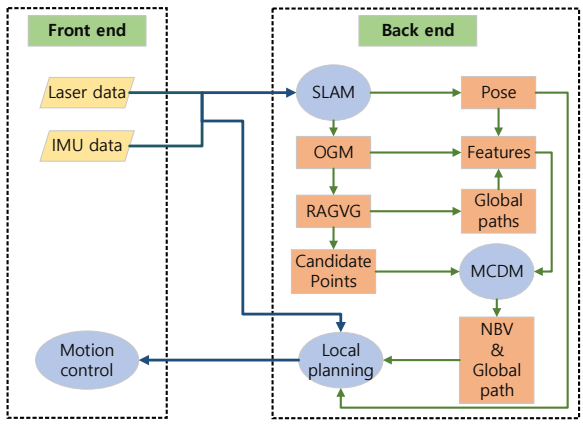

Figure 9. Mobile robot exploration framework.

In the previous section, the solutions of step (1) and (2) have already been presented. This section will illustrate the employed methods for the remaining steps, including how to select the NBV from CPs using the MCDM approach and how to quickly find a global path from the location of the robot to the NBV.

\subsection{Features of CPs}


To determine the NBV, each $\mathrm{CP} p_{i} \in S$ has to be evaluated according to some features. All the features taken into consideration constitute the feature set $F$. The utility $u_{j}\left(p_{i}\right)$ is calculated with respect to the $j$-th feature of $p_{i}$ according to certain rules, assuming that $u_{j}\left(p_{i}\right) \in[0,1]$ and that the larger the utility is, the better the CP. Thus, naturally, if there are $n$ features in $F, p_{i}$ can be measured with a utility vector $U\left(p_{i}\right)=$ $\left(u_{1}\left(p_{i}\right), u_{2}\left(p_{i}\right), \ldots, u_{n}\left(p_{i}\right)\right)$.

In the proposed exploration strategy, a $\mathrm{CP} p$ is evaluated according to the following features: (1) $A(p)$ : the estimation of the unexplored grids that would be sensed from $p$; (2) $D(p)$ : the length of the global path from the location of the robot to $p$; (3) $T(p)$ : the sum of the turning angles of the global path; (4) $C(p)$ : the coverage condition of $p$.

Specifically, $A(p)$ is computed using a casting approach within five metres. This approach simulates casting a 5-metre single laser ray at intervals of $\pi / 180$ from $p$, and estimates the potential information gain by counting the sum of unexplored grids that can be sensed by these 360 laser rays. $D(p)$ is the length of the global path; it is calculated by the sum of Euclidean distances of the sampling points of the global path. The other feature $T(p)$ describes the complexity of the global path with the sum of the turning angles.

$C(p)$ is used to determine whether $p$ has been sensed, which is important information for avoiding omission and repetition. The estimation of $C(p)$ depends on the specialty of the external sensor, which determines the updating on the coverage occupancy map (COM). A COM estimates the group of grids that have been detected by specific sensors. Figure 10 shows a COM updated by a 3D LRF whose field of view is $2 \pi$. With a given COM, if $C G$ is established as the set of covered grids, then $C(p)$ can be computed according to a distance function as Eq.(10).

$$
\begin{array}{r}
x_{u c}=\operatorname{argmin}_{x \notin C G} \operatorname{Dist}(p, x), \\
x_{c}=\operatorname{argmin}_{x \in C G} \operatorname{Dist}(p, x), \\
C(p)=\left\{\begin{array}{l}
-\operatorname{Dist}\left(p, x_{u c}\right), \text { if } p \in C G \\
\operatorname{Dist}\left(p, x_{c}\right), \text { if } p \notin C G
\end{array}\right.
\end{array}
$$

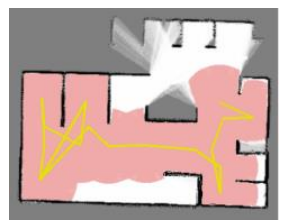

Figure 10. Coverage occupancy map.

These four features work together to determine the "best" CP as the NBV. The utility of each of these features is computed and linearly normalized to $[0,1]$ as Eq.(11).

$$
\begin{gathered}
u_{A}(p)=\frac{A(p)-\min _{q \in C P} A(q)}{\max _{q \in C P} A(q)-\min _{q \in C P} A(q)}, \\
u_{D}(p)=1-\frac{D(p)-\min _{q \in C P} D(q)}{\max _{q \in C P} D(q)-\min _{q \in C P} D(q)^{\prime}}, \\
u_{T}(p)=1-\frac{T(p)-\min _{q \in C P} T(q)}{\max _{q \in C P} T(q)-\min _{q \in C P} T(q)^{\prime}} \\
u_{C}(p)=\frac{C(p)-\min _{q \in C P} C(q)}{\max _{q \in C P} C(q)-\min _{q \in C P} C(q)} .
\end{gathered}
$$

\subsection{MCDM Approach}

An evaluation system must be developed to assign each CP $p \in S$ a score, and then choose the $\mathrm{CP}$ with the highest score as the NBV. Nevertheless, a multi-dimensional utility vector $U(p)$ cannot be directly used to rank CPs. Hence, the MCDM approach (Basilico, Amigoni, 2011) is employed to evaluate CPs, which provides an aggregation function to define a global utility using a Choquet integral, thus yielding a single value score based on $U(p)$ and the features set $F$.

In MCDM approach, the core is the value of the normalized fuzzy measure function that is defined for a group of features $G \subseteq F$. This function is employed to pre-define the overall contribution of the group of features to the aggregation function according to their dependency relationships. Specifically, the overall contribution of $G$ should be less than the simple sum of the utilities of $G$ that have redundant relationships and should be larger when synergistic relationships exist. Two examples of normalized fuzzy measure function can be found in section 5.2.3.

\subsection{Global Path Planning}

With the RAGVG, it is easy to find a collision-free path from any starting location $L_{s}$ to any other destination location $L_{d}$ using a slight extension of the Dijkstra algorithm. However, Due to the geometrical characteristics of an RAGVD, the global path that consists of the edges of the RAGVG is complex and redundant, as shown in Figure 11(a). Hence, Douglas-Peucker algorithm (Hershberger, Snoeyink, 1992) is employed to simplify the path curve. As shown in Figure 11(b), the simplified path is represented by a series of critical path points, which is more conducive to motion planning instead of continuous pixel chains. In addition, it is obvious that the simplified path is more straightforward than the naive path in terms of time and energy consumption.
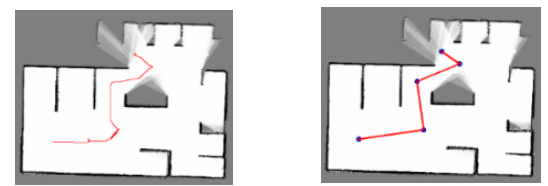

Figure 11. Naive path (left); Simplified path (right)

\section{EXPERIMENTS}

In this section, two categories of experiments will be presented to suggest the validity, practicability and advancement of the proposed method. In section 5.1 we tested the performance of the proposed method for quickly constructing an RAGVG. And in section 5.2 and 5.3 we conducted the proposed RAGVG-based full-coverage exploration framework, in simulations and realworld environment, respectively.

\subsection{Experiment on RAGVG Construction}

5.1.1 Comparative Algorithms, Data and Metrics: From the literature, we found two typical thinning algorithms, including the brushfire algorithm (Choset, Lynch, Hutchinson et al, 2005) and the Zhang-Suen algorithm (Zhang, Suen, 1984). After some minor modifications, their serial and parallel versions are compared with the proposed method.

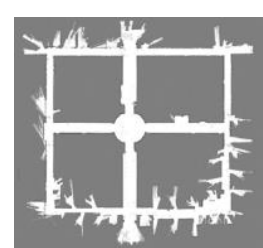

(a) ACES3 of Austin

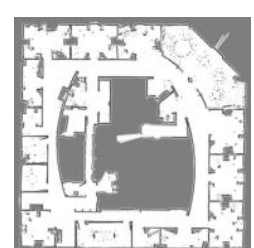

(b) Intel Research Lab
Figure 12. Famous SLAM maps.

Two famous SLAM maps (see Figure 12) are employed to 
compare the performance of the thinning algorithms mentioned above. All of the OGMs are generated by Gmapping-SLAM developed by Cyrill Stachniss et al. (Stachniss, Udo, Grisetti et al, 2018) on two common data sets. The first map contains connecting rooms, rooms on both sides of a long corridor, vertical and parallel corridors, and few clusters. The third map is the hardest one because it is a relatively large indoor space with plentiful clusters that exist in almost every room.

To measure the performance of all the algorithms, some parameters are recorded or computed as metrics, including (1) $T T$, time consumption of the thinning process; (2) $T G$, time consumption of constructing the topological graph; (3) $S N G$, sum of the nodes in the resulting graph; (4) $T L$, total length of the edges in resulting graph and (5) $I R$, information retention of the resulting graph. The information retention, which is computed by comparing a constraint buffer of the GVG and the original OGM, indicates the completeness of a GVG.

5.1.2 Result and Discussion: Ten groups of valid results are collected for each algorithm on each OGM, and the average values of the corresponding metrics are shown in Table 1. The resultant RAGVGs constructed by the proposed method are shown in Figure 13.

\begin{tabular}{|c|c|c|c|c|c|c|c|}
\hline Map & Algorithm & Version & $\begin{array}{c}T T / \\
\mathrm{ms}\end{array}$ & $\begin{array}{c}T G / \\
\mathrm{ms}\end{array}$ & $S N G$ & $T L / \mathrm{p}$ & $\begin{array}{l}I R \\
/ \%\end{array}$ \\
\hline \multirow{3}{*}{1} & Brushfire & $\begin{array}{c}\text { Serial } \\
\text { Parallel }\end{array}$ & $\begin{array}{l}366 \\
327 \\
\end{array}$ & 60 & 149 & 7246 & 99.8 \\
\hline & $\begin{array}{c}\text { Zhang- } \\
\text { Suen }\end{array}$ & $\begin{array}{c}\text { Serial } \\
\text { Parallel }\end{array}$ & $\begin{array}{l}459 \\
151\end{array}$ & 48 & 108 & 5964 & 93.6 \\
\hline & Proposed & $\begin{array}{c}\text { Serial } \\
\text { Parallel }\end{array}$ & $\begin{array}{l}321 \\
130\end{array}$ & 31 & 76 & 4509 & 99.7 \\
\hline \multirow{3}{*}{2} & Brushfire & $\begin{array}{c}\text { Serial } \\
\text { Parallel }\end{array}$ & $\begin{array}{c}1127 \\
912 \\
\end{array}$ & 396 & 468 & 22,781 & 99.8 \\
\hline & $\begin{array}{c}\text { Zhang- } \\
\text { Suen }\end{array}$ & $\begin{array}{c}\text { Serial } \\
\text { Parallel }\end{array}$ & $\begin{array}{c}1943 \\
989\end{array}$ & 324 & 383 & 17,309 & 91.0 \\
\hline & Proposed & $\begin{array}{c}\text { Serial } \\
\text { Parallel }\end{array}$ & $\begin{array}{l}910 \\
539\end{array}$ & 96 & 169 & 12,543 & 99.8 \\
\hline
\end{tabular}

Table 1. Results for constructing RAGVGs.

From the perspective of the time consumption of the thinning process, the proposed algorithm outperforms the other two algorithms for both serial and parallel versions. For the processing of constructing GVGs, the GVDs generated by the brushfire algorithm are extremely redundant, and thus it costs more time to construct a larger topological graph. Furthermore, the GVDs generated by the Zhang-Suen algorithm tend to be fragmented, and thus it costs more time to construct a number of interrupted topological graphs. For information retention, the GVGs constructed by the brushfire and the proposed algorithms covered more than $99 \%$ of the area, but the Zhang-Suen algorithm missed a portion of the area due to its fragmented GVDs.

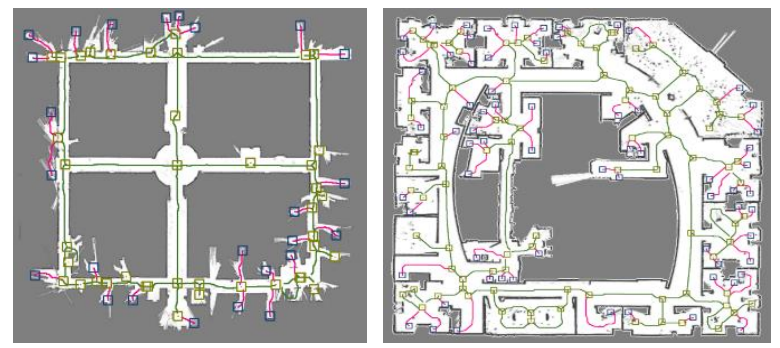

Figure 13. RAGVGs constructed by the proposed algorithm.

It can be clearly seen that the RAGVG constructed by the proposed method can simplify the free area in an OGM into a topological representation. Thus, this RAGVG is detailed to navigate the mobile robot with its nodes and edges. On the one hand, the proposed algorithm can address large-scale OGMs with much less time consumption than other algorithms. On the other hand, the RAGVD generated by the proposed algorithm is much less redundant and non-interrupted.

\subsection{Simulation on Full-Coverage Exploration}

5.2.1 Comparative Method and Metrics: Two tasks, point cloud capture and search and rescue, are used to evaluate the performance of the proposed RAGVG-based autonomous exploration framework. In addition, another popular exploration strategy based on greedily traversing frontiers (Visser, Van Ittersum, Jaime et al, 2007) is used to compare our method.

A number of metrics are used to evaluate the frontier-based method and the proposed method, including (1) $C A$, sum of the coverage area over time; (2) $T T$, total time consumption for finishing exploring the environment; (3) $T N$, time consumption for selecting the NBV in one single decision-making procedure; (4) $S C$, sum of CPs to be evaluated in one single decision-making procedure; and (5) $P C$, percentage of CPs whose global utility is larger than half of the maximum global utility in one single decision-making procedure. In addition, for the search and rescue task, one more metric is referred to as (6) $P F$, sum of trapped people found by the robot over time.

5.2.2 Scenario: An indoor scenario, whose $3 \mathrm{D}$ model is shown in Figure 14, is constructed to simulate autonomous exploration. This indoor scenario involves a $20 \mathrm{~m} \times 20 \mathrm{~m}$ office environment with small rooms, obstacles and a short corridor. For the point cloud capture task, a simulative 3D LRF is employed to collect point clouds. In the search and rescue task, trapped people are randomly distributed in the rooms and can be detected by a simulative biosensor.

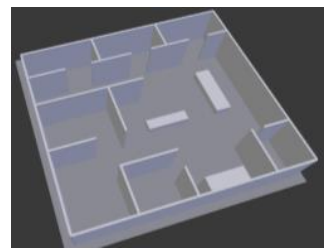

Figure 14. 3D model of the scenario.

5.2.3 Fuzzy Measure Function of MCDM: The specific values of the fuzzy measure function $\mu$ for the two tasks are presented in Table 2, in which $\mu_{l}(G)$ places greater importance on the coverage information and $\mu_{2}(G)$ places greater importance on the potential information gain and distance.

\begin{tabular}{ccc|ccc}
\hline Group of features $G$ & $\mu_{1}(G)$ & $\mu_{2}(G)$ & Group of features $G$ & $\mu_{1}(G)$ & $\mu_{2}(G)$ \\
\hline$A$ & 0.25 & 0.4 & $C, D$ & 0.8 & 0.6 \\
$C$ & 0.4 & 0.15 & $C, T$ & 0.5 & 0.3 \\
$D$ & 0.25 & 0.35 & $D, T$ & 0.3 & 0.4 \\
$T$ & 0.1 & 0.1 & $A, C, D$ & 0.95 & 0.95 \\
$A, C$ & 0.75 & 0.6 & $A, C, T$ & 0.85 & 0.8 \\
$A, D$ & 0.6 & 0.85 & $A, D, T$ & 0.6 & 0.9 \\
$A, T$ & 0.4 & 0.55 & $C, D, T$ & 0.7 & 0.7 \\
\hline
\end{tabular}

Table 2. Values of the fuzzy measure function

5.2.4 Result and Discussion: The results show that all three methods are qualified to complete full-coverage exploration tasks in this indoor space. And the progression of autonomous exploration controlled by MCDM with $\mu_{1}(G), \mu_{2}(G)$ are exhibited in Figure 15, in which the pink pixels represent the coverage area and the yellow polyline indicates the trajectory of the mobile robot.

Table 3 presents some comparisons of the key components in the three methods. The two proposed methods require approximately $20 \%$ less time than the frontier-based method for full-coverage exploration. One of the improvements of the proposed method is that it takes approximately $85 \%$ less time 
than the frontier-based method to select the NBV, which can be partly explained by the $S C$ and $P C$ metrics. On the one hand, the proposed methods' time costs are mainly in constructing an RAGVG from an OGM, whereas the frontier-based method's time cost is mainly in finding the global path with the $A^{*}$ algorithm. On the other hand, the sum of CPs generated from an RAGVG is substantially less than that of the frontier-based method. Overall, compared with the frontier-based method, although the proposed method requires time to construct RAGVGs, the proposed method saves much more time in path finding.

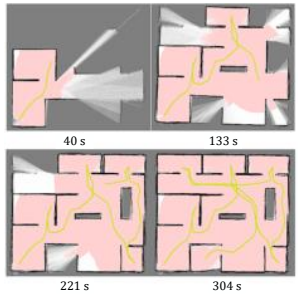

(a)

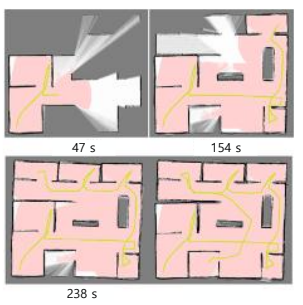

(b)
Figure 15. Simulation progression controlled by (a) $\operatorname{MCDM}-\mu_{l}(G)$ and (b) $\operatorname{MCDM}-\mu_{2}(G)$

\begin{tabular}{cccccc}
\hline Methods & & $T T / \mathrm{s}$ & $T N / \mathrm{ms}$ & $S C$ & $P C / \%$ \\
\hline \multirow{3}{*}{ MCDM- $\mu_{I}(G)$} & Max & 332 & 182 & $\mathbf{8}$ & 79.3 \\
& Average & 320 & 163 & $\mathbf{5 . 1}$ & $\mathbf{4 8 . 1}$ \\
& Min & 304 & 138 & $\mathbf{3}$ & $\mathbf{2 4 . 8}$ \\
\hline \multirow{3}{*}{ MCDM- $\mu_{2}(G)$} & Max & $\mathbf{3 2 7}$ & $\mathbf{1 7 1}$ & 9 & $\mathbf{8 1 . 7}$ \\
& Average & $\mathbf{3 1 9}$ & $\mathbf{1 6 2}$ & 5.4 & 46.2 \\
& Min & $\mathbf{3 0 2}$ & $\mathbf{1 2 5}$ & $\mathbf{3}$ & 19.7 \\
\hline \multirow{3}{*}{ Frontier-based } & Max & 419 & 1370 & 26 & 38.5 \\
& Average & 392 & 1258 & 22.0 & 23.2 \\
& Min & 375 & 943 & 18 & 12.1 \\
\hline
\end{tabular}

Table 3. $T T$ and $T N$ values for the simulations

Coverage in Figure 16 represents the area of the indoor space scanned by 3D LRF, and in Figure 17, the trapped people found represent how many people the mobile robot found in the simulated building. The results clearly show that the task processes controlled by the two proposed methods are better than that of the frontier-based method.

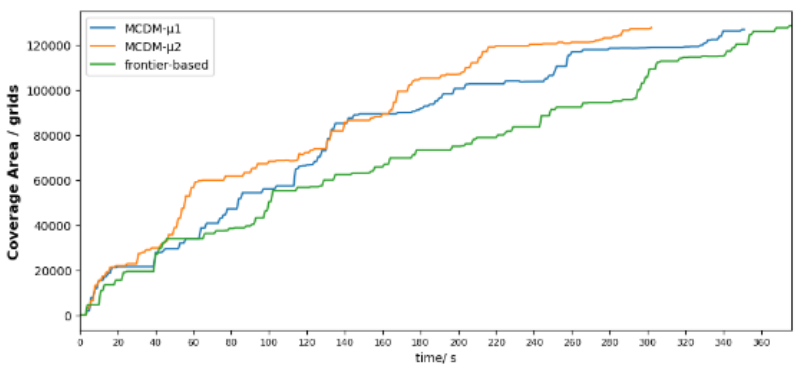

Figure 16. Coverage

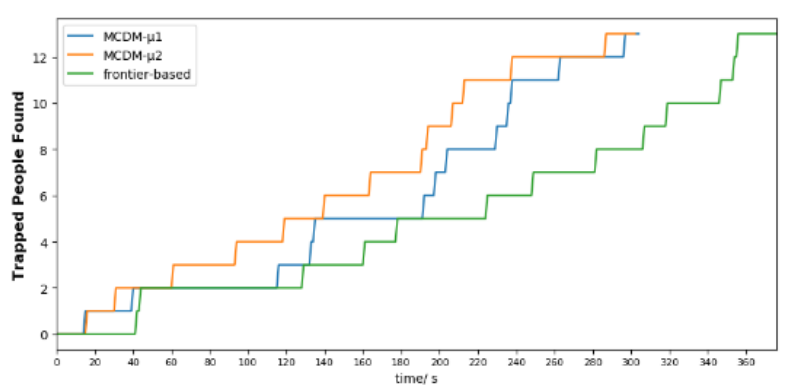

Figure 17. Trapped people found

\subsection{Experiment in a Real-world Museum}

The proposed method was also implemented on a real mobile robot, for capturing the point clouds of a real-world museum.

5.3.1 Scenario and Equipments: The real-world scenario is a 45 $\mathrm{m} \times 27 \mathrm{~m}$ indoor museum that lies on the left side of the building in Figure 18(a). More intuitively, one part of the interior and a 3D indoor model of the museum is respectively shown in Figure 18(b) and (c).

As is shown in Figure 19, our mobile robot is equipped with two 2D LRFs, the scan range of each of them is $30 \mathrm{~m}, 270^{\circ}$. The fixed LRF is used for SLAM while the rotating LRF is used for capturing $3 \mathrm{D}$ point clouds. At each step, the mobile robot stops at the location of NBV, and it takes 12 seconds to finish capturing at this station.
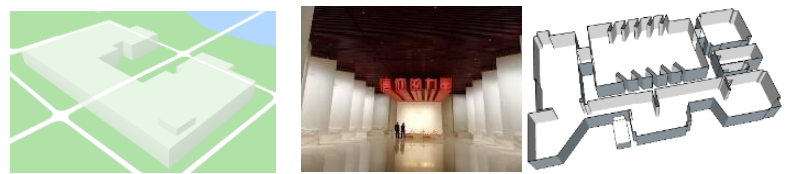

Figure 18. 2.5D building model (left); Part of the interior of the museum (mid); 3D indoor model of the museum (right)

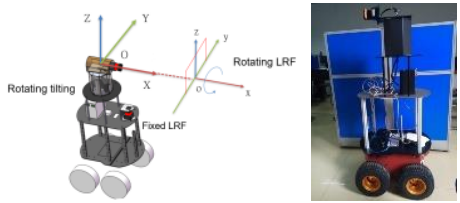

Figure 19. The design of our robot (left); our real robot (right)

5.3.2 Result and Discussion: The progression of the experiment of point cloud capture task in the real-world museum is shown in Figure 20. It took 576 seconds to finish capturing 15 station point clouds, which indicates that the mobile robot stopped more than 180 seconds. Hence, it only took less than 396 seconds to finish full-coverage exploration in this museum. The resulting point clouds is shown in Figure 21, and it can be obviously seen that there barely exists missing part in the integrated point clouds. And it can be claimed that the proposed method also performs well for real-world scenario and real robot.

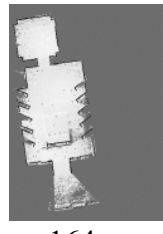

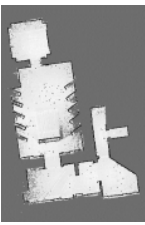

$339 \mathrm{~s}$

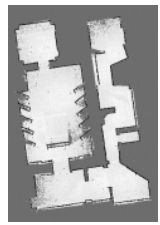

$482 \mathrm{~s}$

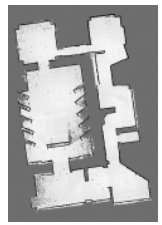

$576 \mathrm{~s}$
Figure 20. Autonomous exploration controlled by $\operatorname{MCDM}-\mu_{2}(G)$

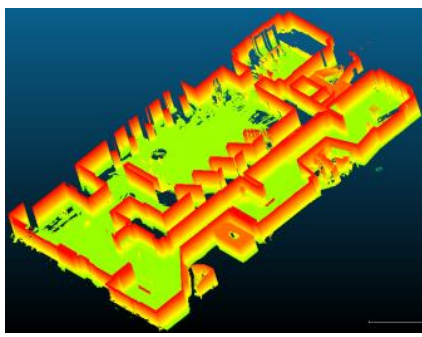

Figure 21. The resulting point clouds after removing the roof

\section{CONCLUSIONS}

Autonomous exploration is an important ability of mobile robotics, and the main aim of research on this topic is to guide a robot to explore a previously unknown space while consuming 
less time. This paper presents a novel autonomous exploration system based on an RAGVG, and the discovered improvements are as follows:

-- A fast, robust and parallel algorithm was proposed for constructing an RAGVG from an OGM;

-- The efficiency of selecting the NBV was markedly improved by generating more competitive CPs and by using fast graphbased path planning;

-- The number of local obstacles that must be avoided was reduced by means of a collision-free global path that is rapidly generated from an RAGVG.

Simulation and real-world experiments show that the proposed algorithm can generate an RAGVD quickly and robustly that is one-pixel-wide, non-interrupted and relatively non-redundant, and the RAGVG constructed from this RAGVD can represent almost all the connectivity of an indoor space with fewer edges and nodes. Selecting the NBV based on an RAGVG takes $85 \%$ less time than the frontier-based method. Combining the improvements mentioned above, the total time consumption for a full-coverage exploration by the proposed method is approximately $20 \%$ less than that by the frontier-based method.

However, some factors still exist that affect the efficiency of autonomous exploration. From the perspective of graph theory, the RAGVGs are still redundant and can be further simplified. In terms of the MCDM approach, the values of the fuzzy measure function are empirical. These topics should be explored further.

Acknowledgments: This study is funded by the National Key R\&D Program of China (2017YFB0503701) and the National Natural Science Foundation of China (41871298).

\section{REFERENCES}

Burgard W, Moors M, Stachniss C, et al, 2005: Coordinated multi-robot exploration[J]. IEEE Transactions on robotics, 2005, 21(3): 376-386.

Grisetti G, Stachniss C, Burgard W, 2007: Improved techniques for grid mapping with rao-blackwellized particle filters[J]. IEEE transactions on Robotics, 2007, 23(1): 34-46.

Thrun S, 1998: Learning metric-topological maps for indoor mobile robot navigation[J]. Artificial Intelligence, 1998, 99(1):21-71.

Kulich M, Kubalík J, Přeučil L, 2019: An Integrated Approach to Goal Selection in Mobile Robot Exploration[J]. Sensors, 2019, 19(6): 1400

Stachniss C, Burgard W, 2003: Mapping and exploration with mobile robots using coverage maps[C]. Intelligent Robots and Systems, 2003. (IROS 2003). Proceedings. 2003 IEEE/RSJ International Conference on. IEEE, 2003, 1: 467-472.

Yamauchi B, 1997: A frontier-based approach for autonomous exploration[C]. Computational Intelligence in Robotics and Automation, 1997. CIRA'97., Proceedings., 1997 IEEE International Symposium on. IEEE, 1997: 146-151.

González-Banos H H, Latombe J C, 2002: Navigation strategies for exploring indoor environments $[\mathrm{J}]$. The International Journal of Robotics Research, 2002, 21(10-11): 829-848.

Visser A, Van Ittersum M, Jaime L A G, et al, 2007: Beyond frontier exploration[C]. Robot Soccer World Cup. Springer, Berlin, Heidelberg, 2007: 113-123.

Stachniss C, Grisetti G, Burgard W, 2005: Information gainbased exploration using rao-blackwellized particle filters[C]. Robotics: Science and Systems. 2005, 2: 65-72.

Carrillo H, Dames P, Kumar V, et al, 2015: Autonomous robotic exploration using occupancy grid maps and graph SLAM based on Shannon and Rényi entropy[C]. 2015 IEEE international conference on robotics and automation (ICRA). IEEE, 2015: 487-494.

Carrillo H, Dames P, Kumar V, et al, 2018: Autonomous robotic exploration using a utility function based on Rényi's general theory of entropy[J]. Autonomous Robots, 2018, 42(2): 235-256.

Calisi D, Farinelli A, Iocchi L, et al, 2005: Autonomous navigation and exploration in a rescue environment[C]. Safety, Security and Rescue Robotics, Workshop, 2005 IEEE International. IEEE, 2005: 54-59.

Basilico N, Amigoni F, 2011: Exploration strategies based on multi-criteria decision making for searching environments in rescue operations[J]. Autonomous Robots, 2011, 31(4): 401.

Low K L, Lastra A, 2006: An adaptive hierarchical next-bestview algorithm for $3 \mathrm{~d}$ reconstruction of indoor scenes[C]. Proceedings of 14th Pacific Conference on Computer Graphics and Applications (Pacific Graphics 2006). 2006: 1-8.

Quintana B, Prieto S A, Adán A, et al, 2016: Semantic scan planning for indoor structural elements of buildings[J]. Advanced Engineering Informatics, 2016, 30(4): 643-659.

Takahashi O, Schilling R, 1989: Motion planning in a plane using generalized Voronoi diagrams[J]. IEEE Transactions on Robotics and Automation, 1989, 5(2):150

Howie Choset J B, 1995: Sensor Based Planning, Part I: The Generalized Voronoi Graph[C]. IEEE International Conference on Robotics \& Automation. IEEE, 1995.

Nagatani K, Choset H, 1999: Toward robust sensor-based exploration by constructing reduced generalized Voronoi graph[C]. IEEE/RSJ International Conference on Intelligent Robots \& Systems. IEEE, 1999.

Choset, H, 2000: Sensor-Based Exploration: The Hierarchical Generalized Voronoi Graph[J]. The International Journal of Robotics Research, 2000, 19(2):96-125.

Lau B, Sprunk C, Burgard W, 2010: Improved updating of Euclidean distance maps and Voronoi diagrams[C]. Intelligent Robots and Systems (IROS), 2010 IEEE/RSJ International Conference on. IEEE, 2010: 281-286.

Tsardoulias E G, Serafi A T, Panourgia M N, et al, 2014: Construction of minimized topological graphs on occupancy grid maps based on GVD and sensor coverage information[J]. Journal of Intelligent \& Robotic Systems, 2014, 75(3-4): 457-474.

Tsardoulias E G, Iliakopoulou A, Kargakos A, et al, 2017: CostBased Target Selection Techniques Towards Full Space Exploration and Coverage for USAR Applications in a Priori Unknown Environments[J]. Journal of Intelligent \& Robotic Systems, 2017, 87(2): 313-340. 
Tsardoulias E G, Iliakopoulou A, Kargakos A, et al, 2016: A review of global path planning methods for occupancy grid maps regardless of obstacle density[J]. Journal of Intelligent \& Robotic Systems, 2016, 84(1-4): 829-858.

Valero-Gomez A, Gomez J V, Garrido S, et al, 2013: The path to efficiency: Fast marching method for safer, more efficient mobile robot trajectories[J]. IEEE Robotics \& Automation Magazine, 2013, 20(4): 111-120.

Saeed K, Rybnik M, Tabedzki M, 2001: Implementation and advanced results on the non-interrupted skeletonization algorithm[C]. International Conference on Computer Analysis of Images and Patterns. Springer, Berlin, Heidelberg, 2001: 601609 .

Saeed K, Tabędzki M, Rybnik M, et al, 2010: K3M: A universal algorithm for image skeletonization and a review of thinning techniques[J]. International Journal of Applied Mathematics and Computer Science, 2010, 20(2): 317-335.

Zhang T Y, Suen C Y. Suen, C.Y., 1984: A Fast Parallel Algorithm for Thinning Digital Patterns. Communications of the ACM 27(3), 236-239[J]. Communications of the ACM, 1984, 27(3):236-239.

Choset H, Lynch K, Hutchinson S, et al, 2005: Principles of robot motion: Theory, algorithms, and implementations. MIT Press, Cambridge, MA[J]. Proceedings of the Society for Experimental Biology \& Medicine Society for Experimental Biology \& Medicine, 2005, 147(1):512-512.

Hershberger J E, Snoeyink J, 1992: Speeding up the DouglasPeucker Line-Simplification Algorithm[M]. University of British Columbia, Department of Computer Science, 1992.

Cyrill Stachniss, Udo Frese, Giorgio Grisetti, et al, 2018: https://openslam-org.github.io/. 